\title{
Los ácidos carboxílicos de extractos vegetales y la humedad del suelo influyen en la producción y el rajado del fruto de uchuva (Physalis peruviana L.)
}

\section{Carboxylic acids of plant extracts and soil humidity influence production and cracking of cape gooseberry (Physalis peruviana L.) fruit}

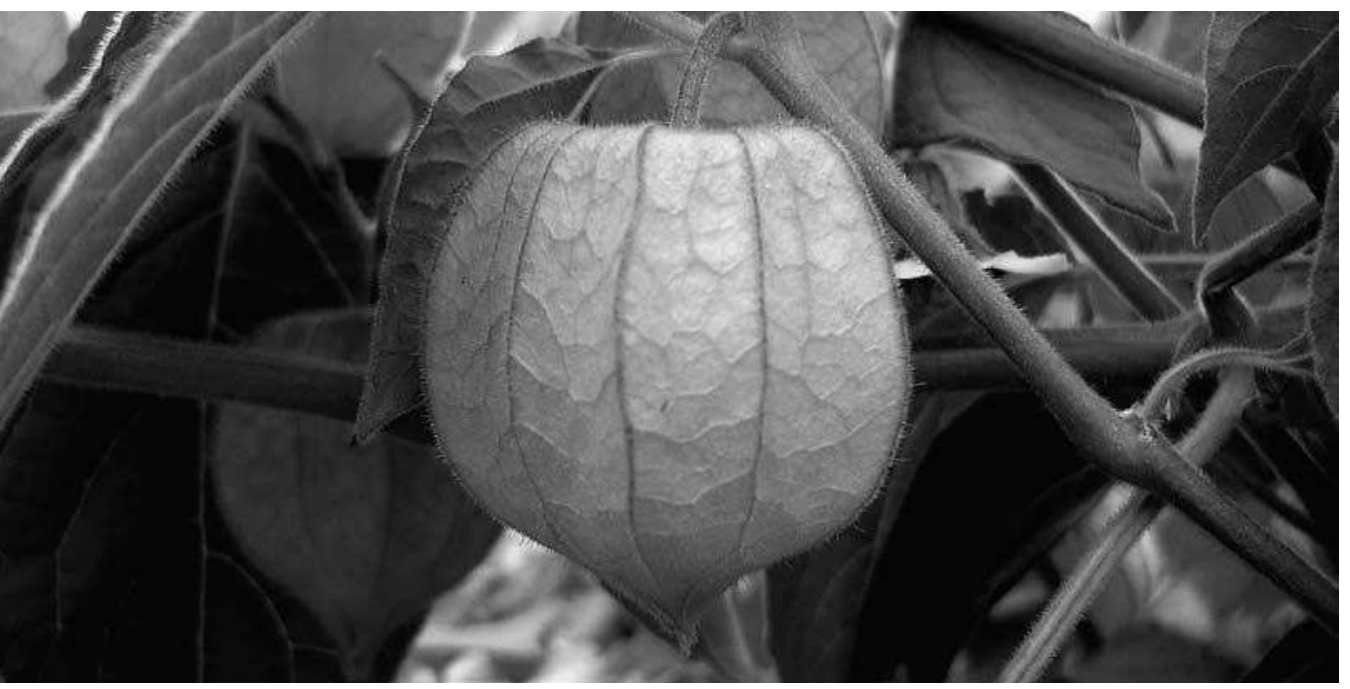

BADIR GUERRERO'

MAURICIO VELANDIA²

GERHARD FISCHER ${ }^{3}$

HUGO MONTENEGRO4

Fruto de uchuva maduro en su cáliz.

Foto: G. Fischer

\section{RESUMEN}

Con el fin de disminuir el porcentaje del rajado y aumentar tamaño y peso del fruto de uchuva, se estudió el efecto de los ácidos carboxílicos, contenidos en el producto Carboxy® Calhard (ácidos poli-hidroxi-carboxílicos [78 $\left.\mathrm{g} \cdot \mathrm{L}^{-1}\right]$, adicionados con calcio y boro), en dosis de 0, 1,5 y 3,0 L $\cdot \mathrm{ha}^{-1}$ a tres niveles de humedad en el suelo, $10-25 \%, 25-50 \%$ y $50-75 \%$, en un cultivo comercial en el municipio de Chocontá (Cundinamarca). Los tratamientos con 0, 1,5 y 3,0 L ha ha de Calhard a 50-75\% de humedad evidenciaron el porcentaje más alto de frutos de exportación, 40,17, 41,67 y 45,33\% respectivamente, de la misma forma menor porcentaje de frutos para el mercado nacional $(31,33,29,67$ y $37,0 \%$, respectivamente) debido a un mayor contenido de humedad en el suelo. Los tratamientos 0 y 1,5 L/ha ${ }^{-1}$ de Calhard a 50-75\% humedad obtuvieron el mayor porcentaje de frutos rajados, 28,50 y $28,67 \%$, respectivamente, mientras que $3,0 \mathrm{~L} \cdot \mathrm{ha}^{-1}$ de Calhard a $50-75 \%$ de humedad redujo significativamente el porcentaje de frutos rajados, con $17,67 \%$, debido a la aplicación de ácidos carboxílicos. El peso fresco del fruto sin cáliz se relacionó con la dosis del producto y la humedad del suelo mostrando el

Ingeniero Agrónomo, Facultad de Agronomía, Universidad Nacional de Colombia, Bogotá. badirguerrero@gmail.com Ingeniero Agrónomo, Facultad de Agronomía, Universidad Nacional de Colombia, Bogotá. mavelandial@unal.edu.co Profesor Asociado, Facultad de Agronomía, Universidad Nacional de Colombia, Bogotá. gerfischer@gmail.com Profesor Asociado, Facultad de Agronomía, Universidad Nacional de Colombia, Bogotá. hmontenegrog@gmail.com 


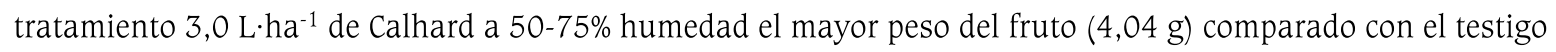
(3,58 g). La aplicación de Calhard, 1,5 y 3,0 L ha ha ${ }^{-1}$, aumentó el diámetro ecuatorial del fruto, con valores de 18,56 y $18,95 \mathrm{~mm}$, respectivamente, mientras que el tratamiento $0 \mathrm{~L} \cdot \mathrm{ha}^{-1}$ de Calhard y el testigo mostraron un diámetro menor $(18,39 \mathrm{~mm})$.

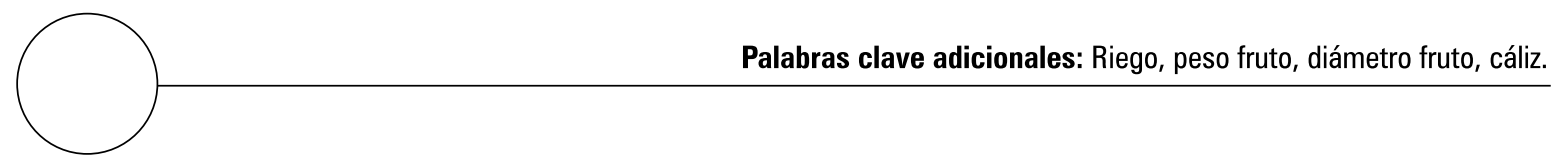

\section{ABSTRACT}

In order to reduce percentage of fruit cracking and increase size and weight of cape gooseberry fruits, the effect of carboxylic acids contained in the product Carboxy® Calhard (poly-hydroxy-carboxylic acids $\left[78 \mathrm{~g} \cdot \mathrm{L}^{-1}\right]$, complemented with calcium and boron) in a commercial crop was studied. Product doses of $0,1,5$ and 3,0 $\mathrm{L} \cdot \mathrm{ha}^{-1}$ and three levels of soil humidity: 10-25, 25-50, and 50-75\% were used. The treatments $0,1,5$, and $3 \mathrm{~L} \cdot \mathrm{ha}^{-1}$ Calhard at 50-75\% soil humidity resulted in a highest percentage of exportable fruits $40,17,41,67$, and $45,33 \%$ respectively, likewise the lowest number of fruits for the national market 31,33, 29,67 and 37,0\%, respectively, due to the high soil humidity. The treatments 0 and $1,5 \mathrm{~L} \cdot \mathrm{ha}^{-1}$ Calhard at $50-75 \%$ soil humidity showed the highest percentage of cracked fruits, 28,50 and $28,67 \%$ respectively, whereas $3 \mathrm{~L}$-ha- ${ }^{-1}$ Calhard at $50-75 \%$ soil humidity reduced significantly the percentage of cracked fruits to $17,67 \%$ due to the application of carboxylic acids. Fruit fresh weight without calyx was related to the product dose and soil humidity, showing the treatment 3,0 L-ha-1 Calhard at 50-75\% soil humidity as the one with the highest fruit fresh weight $(4,04 \mathrm{~g})$ compared to the control $(3,58 \mathrm{~g})$. Calhard application of 1,5 and 3,0 L:ha-1 increased the equatorial diameter of fruits with values of 18,56 and $18,95 \mathrm{~mm}$, respectively, while $0 \mathrm{~L} \cdot \mathrm{ha}^{-1}$ Calhard and the control showed the less fruit diameter values $(18,39 \mathrm{~mm})$.

Additional key words: Irrigation, fruit weight, fruit diameter, calyx.

$\longrightarrow$

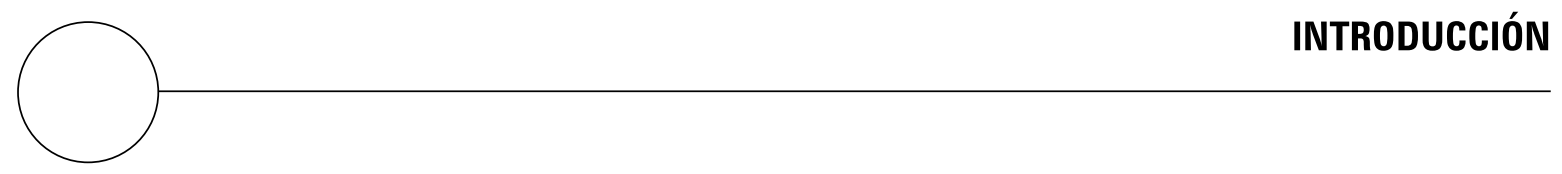

La uchuva (Physalis peruviana L.) pertenece a la familia de las solanáceas y es originaria de los Andes. En Colombia, su producción se encuentra en altitudes entre los 1.800 y $2.800 \mathrm{msnm}$ con temperaturas promedios entre 13 y $18^{\circ} \mathrm{C}$ (Fischer y Angulo, 1999). Es el fruto promisorio más ex- portado de Colombia (\$US 23.841.000 en 2005) con un área sembrada de 7.290 ha y una producción de 11.327 .6 t en 2004 (Ministerio de Agricultura y Desarrollo Rural, 2006). En el País, el cultivo de uchuva ha aumentado en promedio $35 \%$ del área sembrada desde 2000 a 2004; en 2003 
el mayor productor fue el departamento Cundinamarca con 273,5 ha, equivalentes al $75,9 \%$ del total del área sembrada en el País; siguen Boyacá con 37,4 ha (10,4\%), Antioquia con 18,3 ha $(5,0 \%)$ y otros departamentos con 31,3 ha $(8,7 \%)$ (Sanabria, 2005).

El fruto de la uchuva es una baya jugosa y carnosa con altos niveles de Fe y P, vitaminas A y C y fibra (Fischer, 2000) de color amarillo-naranja cuando está maduro; se desarrolla dentro del cáliz gamosépalo globoso acrecente que cubre el fruto hasta madurez. El cáliz, formado por cinco sépalos protege al fruto contra insectos, pájaros, enfermedades y condiciones climáticas extremas (radiación, cambios bruscos de temperatura, lluvias fuertes y granizadas); sirve como una fuente de carbohidratos durante los primeros 20 días del crecimiento del fruto (Fischer y Lüdders, 1997) y prolonga la vida poscosecha en dos terceras partes (Herrera, 2000).

La uchuva se cultiva en Colombia en el interior del país donde se presenta una precipitación bimodal que hace que en algunos meses sea necesario el riego para corregir déficit hídrico temporal (Campos, 2000). La evapotranspiración, el agua de riego y la percolación son parte de los componentes para la estimación de la necesidad de riego, factor importante en la producción agrícola, que influye en el rajado del fruto (Torres et al., 2004).

El rajado es un término general que ha sido aplicado a ciertos desórdenes fisiológicos de las frutas, expresado como fractura en la cutícula o epidermis del fruto (Fischer, 2005). Se ha atribuido principalmente al balance hídrico de la planta que condiciona el crecimiento y fisiología de las hojas, tallos y raíces, afectando la velocidad del crecimiento y la producción de frutos por el aporte de asimilados, utilizados tanto para el crecimiento, como para el almacenamiento y al ser la expansión del fruto el resultado de la acumulación de agua en la pulpa (Grange, 1993).
El fruto de la uchuva tiene cierta predisposición al rajado, por ser jugoso, tener epidermis muy delgada, y por el contenido de semillas (100300) que posiblemente por influencias hormonales atraen buenas cantidades de agua y carbohidratos (Fischer, 2005). En tomate, planta pariente de la uchuva, se concluyó que la epidermis de la fruta es susceptible al rajado durante el proceso de maduración y a los cambios de humedad del suelo que pueden causar una reducción de la fuerza y elasticidad de la epidermis (Peet, 1992). El agrietado, es menos severo cuando hay una irrigación continua; por esto, es conveniente aplicar riego para evitarlo (Peet y Willits, 1995).

El calcio se encuentra presente como parte de la estructura orgánica (Rodríguez, 1992); mantiene la estructura y características propias de permeabilidad de las membranas dando rigidez a la célula y su contenido aumenta con la edad (Stutte, 1995). El calcio se encuentra principalmente en la pared celular formando sales insolubles al reaccionar con los ácidos de la lámina media, la cual está formada de pectatos de calcio y entra en el metabolismo de formación del núcleo y mitocondrias (Street, 1969; Rojas y Rovalo, 1985). Además, forma parte del sistema amortiguador en la savia de la planta y en componentes de los pelos radicales en una acción directa (Uvalle, 1992). El calcio está gradualmente asociado a los procesos de maduración de frutos y vida de almacenamiento poscosecha. Concentraciones altas de calcio en los tejidos del fruto resultan en una tasa lenta de maduración, cantidades más bajas de respiración y producción reducida de etileno (Ferguson, 1979). Por otro lado, Watkins y Ferguson (1981) mencionan que el principal sitio para la acción del calcio en senescencia y maduración puede estar en la estructura y función de las membranas y en la estructura de la pared celular, aunque altas concentraciones externas de calcio son una ventaja en reducir la tasa de senescencia o madurez, Shear (1971) encontró que 
las deficiencias en calcio y boro pueden provocar el desarrollo de frutos rajados.

El boro es el tercer elemento involucrado con el rajado del fruto (Cooman et al., 2005), y es importante para la formación de paredes celulares debido a que es necesario para la síntesis de pectinas (Salisbury y Ross, 1994). Las plantas adsorben el boro principalmente bajo la forma de ácido bórico sin disociar $\mathrm{H}_{3} \mathrm{BO}_{4}$ representado con mayor exactitud como $\mathrm{B}[\mathrm{OH}]_{3}$. Dentro de la planta el boro es poco móvil (Salisbury y Ross, 1994). Gordillo et al. (2004) encontraron que una fertilización baja en boro aumenta la incidencia del rajado. La utilización del boro ha sido reportada para el control del rajado en frutas y vegetales por Kienholz (1942), Dube et al. (1969), Pierzon et al. (1971) y Raese (1989).

Cooman et al. (2005) realizaron un estudio para determinar el efecto de la oferta del calcio, boro y cobre sobre el rajado del fruto de uchuva dando como resultado un aumento en el rendimiento de la cosecha debido a la adición de calcio y cobre. En las variables peso fresco y rajado de fruto, el boro y el calcio mostraron diferencias significativas, aumentando el peso y reduciendo el número de frutos rajados.

Variando el balance hídrico en uchuva, se mostró que los frutos que han sufrido un estrés severo por déficit de agua en cualquier etapa del cultivo, son de menor tamaño y peso y tienden a presentar un rajado severo (Torres et al., 2004), estos autores concluyeron que las plantas de uchuva que sufren estrés hídrico durante las primeras semanas de su desarrollo, son más afectadas por el rajado, tienen frutos más pequeños (encontrándose en la fase de división celular) debido a una posible reducción mayor de elasticidad de la epidermis, que aquellas que son sometidas a una sequía durante la segunda fase de desarrollo del fruto.

Gordillo et al. (2004) estudiaron el efecto de tres láminas de riego (sin; 80, 100 y 120\% de la eva- poración) y la fertilización sobre la incidencia del rajado en frutos de uchuva en la zona de Silvana (Cundinamarca). El menor porcentaje de rajado ocurrió en el tratamiento en el cual no se aplicó riego, mientras la fertilización técnica sin Boro y la tradicional del agricultor fueron los que mayor porcentaje de frutos rajados presentaron. El testigo (sin fertilización) arrojó el menor porcentaje de frutos rajados, y también el menor porcentaje de rendimiento.

Rodríguez y Pinzón (2000) evaluaron el efecto del polímero absorbente (de agua) Stockosorb sobre la producción y calidad del fruto de uchuva en el municipio El Rosal (Cundinamarca) y encontraron que las dosis de 10, 20 y $30 \mathrm{~g} /$ planta del producto produjeron frutos de 9,8, 10,0 y 10,0 g comparado con solamente $7,8 \mathrm{~g}$ del testigo. Además, las dosis de $20 \mathrm{~g}$ Stockosorb/planta aumentaron el rendimiento de frutos de $2,30 \mathrm{~kg}$ (del testigo) a $2,88 \mathrm{~kg} /$ planta. Con referencia al rajado, la dosis de $10 \mathrm{~g} /$ planta Stockosorb bajó el número de frutos rajados de 41 (del testigo) a 33 frutos/planta.

De acuerdo con Innovak Colombia Ltda. (2006), los ácidos carboxílicos son compuestos de 2 a 8 carbonos en su estructura básica, con un peso molecular menor a $200 \mathrm{~g} / \mathrm{mol}$ aniónico, polihidroxilados y policarboxílicos. Estas características les permiten tener una amplia movilidad en el suelo y la planta; así como influir en los procesos de asimilación de nutrientes y en procesos del metabolismo de carbohidratos de las plantas necesarios para su normal crecimiento y desarrollo.

Según investigaciones en la Universidad de Arkansas, reportado por Innovak Ltda. (2006), hay evidencia, que los ácidos polihidrocarboxílicos actúan dentro de la planta de la siguiente forma: (a) Aumentan la síntesis de metabolitos de transporte (polioles), favoreciendo el movimiento basípeto de auxinas y fotosintatos aportadores de estructuras de carbono hacia la raíz. (b) Favorecen el transporte de nutrientes hacia los sitios de de- 
manda. (c) Incrementa la actividad respiratoria, lo cual permite disponer de mayor cantidad de energía en las células de la raíz para la absorción de nutrientes. (d) Induce una síntesis de novo de ácidos carboxílicos, actuando estos como agentes quelatantes de muchos micro elementos. (e) La mayor actividad respiratoria, es soportada por un incremento en la actividad fotosintética provocando una mayor acumulación de biomasa. (f) Intensifican el bombeo electrogénico a nivel de las membranas celulares, representado en un mayor ingreso por unidad de raíz.

La importancia de los ácidos carboxílicos está en su relación con el metabolismo del nitrógeno. Estos ácidos contribuyen en la formación de casi todos los tejidos, son componentes esenciales en muchas sustancias vegetales de trascendencia; se encuentran en las vitaminas que actúan como grupos funcionales de las enzimas importantes en la respiración en molécula de los ácidos nucleicos y en los alcaloides (Kirk, 1962). Aun más importante el nitrógeno vegetal que forma parte de las moléculas proteínicas, las cuales intervienen en el mecanismo enzimático que hace posible la realización del metabolismo celular (Kirk, 1962; Salisbury y Ross, 1994).

El objetivo de este trabajo fue evaluar la influencia de los ácidos carboxílicos, adicionados con calcio y boro, siendo un producto todavía no evaluado en el cultivo de la uchuva en Colombia, a tres niveles de humedad en el suelo, con el fin de estudiar estas variables en el rajado y peso del fruto.

\section{MATERIALES Y MÉTODOS}

Este trabajo se realizó en la finca Los Arrayanes, municipio de Chocontá (Cundinamarca) a una altitud de $2.650 \mathrm{msnm}$, temperatura media de $14,0^{\circ} \mathrm{C}$ y $1.500 \mathrm{~mm}$ de precipitación promedio anual. Se seleccionaron plantas de uchuva, ecotipo Colombia sembradas el 1 de Febrero de 2005.
El experimento inició en marzo de 2006 con la primera aplicación del producto y el suministro de riego, con los tratamientos como indica la tabla 1, en un diseño experimental de arreglo factorial $3 \times 3$ con 3 repeticiones.

(A) Humedad del suelo en los siguientes contenidos: $10-25 \%, 25-50 \%$ y $50-75 \%$. Las mediciones de humedad en el suelo se hicieron con un tensiómetro los días lunes y jueves durante el experimento, realizando el correspondiente ajuste mediante la aplicación de riego al suelo.

(B) Dosis del producto Carboxy Calhard (ácidos poli-hidroxi-carboxílicos $\left(78 \mathrm{~g} \cdot \mathrm{L}^{-1}\right)$, determinados como carbono $\left(102,78 \mathrm{~g} \cdot \mathrm{L}^{-1}\right)$, calcio elemental $\mathrm{CaO}$ $\left(140,5 \mathrm{~g} \cdot \mathrm{L}^{-1}\right)$, boro elemental B $\left(3,5 \mathrm{~g} \cdot \mathrm{L}^{-1}\right)$ de la empresa Innovak. Las dosis suministradas fueron 0, 1,5 y 3,0 L'ha-1. Las aplicaciones al follaje se realizaron mediante una bomba de espalda de 20 L (boquilla de cono hueco 8005 con un ángulo de $80^{\circ} \mathrm{y}$ tasa de flujo de $0,50 \mathrm{gal} \cdot \mathrm{min}^{-1}$ ) y el producto disuelto en agua.

La unidad experimental estuvo constituida por un área de $2.250 \mathrm{~m}^{2}$, con una distancia entre plantas de 2,5 m y 3,0 m entre surcos, para un total de 300 plantas en el ensayo, distribuidas en 10 plantas por tratamiento con 3 repeticiones en 3 bloques.

Las variables de calidad del fruto evaluadas se clasificaron según NTC 4580 de Icontec (1999) para uchuva, de esta manera se determinaron el peso de la uchuva tipo exportación con y sin cáliz, tipo nacional sin cáliz y rajada sin cáliz y el diámetro ecuatorial de los frutos con las diferentes calidades.

Las evaluaciones se realizaron con una frecuencia de ocho días a partir de la segunda semana de la primera aplicación del producto, el cual se aplicaba con un intervalo de 20 días, tiempo de acción del producto según recomendación empre- 
Tabla 1. Combinaciones de tratamientos en el ensayo.

\begin{tabular}{|c|c|c|c|}
\hline Tratamiento & Dosis Calhard $\left(L \cdot h \mathrm{C}^{-1}\right)$ & Humedad $(\%)$ & \multicolumn{1}{|c|}{ Abreviación } \\
\hline T1 & 0 & $10-25$ & 0 Calh. 10-25\% Hum. \\
\hline T2 & 0 & $25-50$ & 0 Calh. $25-50 \%$ Hum. \\
\hline T3 & 0 & $50-75$ & 0 Calh. $50-75 \%$ Hum. \\
\hline T4 & 1,5 & $25-50$ & 1,5 Calh. $10-25 \%$ Hum. \\
\hline T5 & 1,5 & $25-50$ & 1,5 Calh. $25-50 \%$ Hum. \\
\hline T6 & 1,5 & $50-75$ & 1,5 Calh. $50-75 \%$ Hum. \\
\hline T7 & 3,0 & $25-50$ & 3,0 Calh. $10-25 \%$ Hum. \\
\hline T8 & 3,0 & $25-50$ & 3,0 Calh. $25-50 \%$ Hum. \\
\hline T9 & 3,0 & $50-75$ & 3,0 Calh. $50-75 \%$ Hum. \\
\hline T10 & 0 & 0 & Testigo \\
\hline
\end{tabular}

sa Innovak Ltda., realizando tres aplicaciones en el experimento.

Para cada tratamiento se escogieron 10 plantas por surco con tres réplicas, dejando un borde común entre tratamientos, es decir, un surco sin tratamiento. La cosecha se realizó una vez por semana tomando de cada planta dos frutos al azar, 20 por tratamiento, 600 frutos semanales para un total de 6.000 frutos durante las diez semanas de cosecha.

Para el análisis estadístico se utilizó SAS® (1999) para realizar pruebas de comparación T-student y Tukey y análisis de varianza.

\section{RESULTADOS Y DISCUSIÓN}

\section{Categoría de frutos}

Los tratamientos $0,1,5$ y 3,0 Calh. a 50-75\% Hum. tuvieron el mayor contenido de humedad, obtuvieron los porcentajes de frutos de exportación más altos 40,17, 41,67 y 45,33\% respectivamente (figura 1), asimismo el porcentaje de frutos rajados fue superior a los demás tratamientos $28,50,28,67$ y $17,67 \%$ respectivamente.
Según estos resultados, se puede afirmar que a mayor humedad en el suelo se aumentó tanto el porcentaje de frutos de exportación como el de frutos rajados, para este último, el tratamiento 3,0 Calh. 50-75\% Hum. presentó una reducción de este desorden fisiológico debido al efecto de los ácidos carboxílicos. Este resultado coincide con el estudio realizado por Gordillo et al. (2004) quienes concluyeron que al aplicar mayor cantidad de agua, mayor es el porcentaje de frutos rajados en uchuva.

El menor porcentaje de rajado se presentó en los tratamientos con menor contenido de humedad (0-25\%) con 0, 1,5 y 3,0 Calh. a 10-25\% Hum. y el testigo con valores de 7,83, 4,50, 5,67 y 6,67\% respectivamente. De acuerdo con lo anterior se puede concluir que a menor humedad del suelo se disminuye el porcentaje de frutos rajados y no se hace necesario el uso de ácidos carboxílicos debido a que en el ensayo no se observaron diferencias significativas con niveles bajos de humedad. Gordillo et al. (2004) reportaron que el riego afectó los porcentajes de frutos rajados en los diferentes tratamientos, en la medida que se redujo la cantidad de agua aplicada menor fue el rajado del fruto de uchuva. En general, los frutos de categoría nacional obtuvieron los porcentajes más altos. 


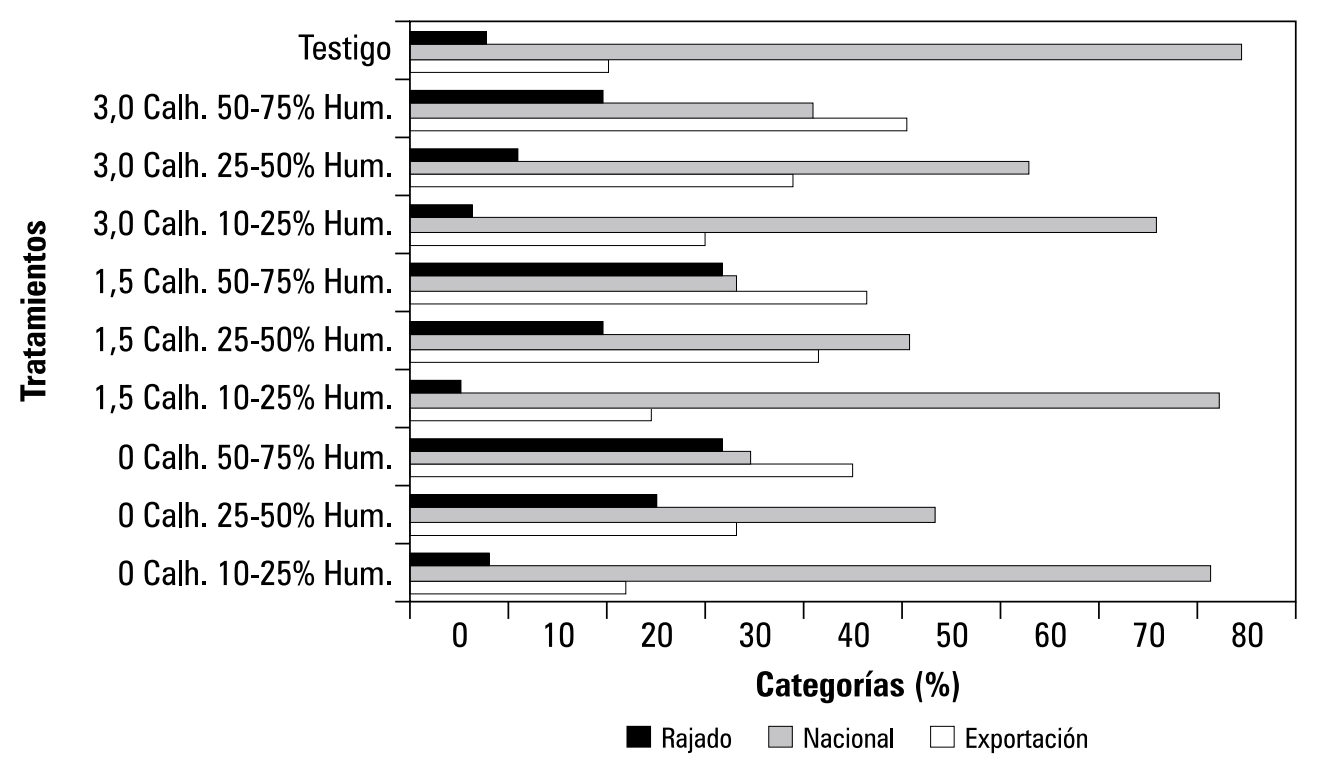

Figura 1. Efecto de tres dosis del producto Calhard $(0,1,5$ y $3 \mathrm{~L} \cdot \mathrm{ha}-1)$ y tres niveles de humedad en el suelo $(10-25,2550$ y 25-75\%) sobre la calidad de los frutos, según norma Icontec NTC 4580 para uchuva.

\section{Peso del fruto sin cáliz}

El análisis de varianza para la variable peso sin cáliz muestra una diferencia significativa entre tratamientos (tabla 2). La prueba T-student para peso sin cáliz y diámetro en la categoría exportación $\operatorname{Pr}>\mathrm{T}<0,0001$, categoría nacional $\operatorname{Pr}>\mathrm{T}<0,0001$, categoría rajada $\operatorname{Pr}>\mathrm{T}<0,0001$ indica una diferencia altamente significativa entre tratamientos. Para las diferentes categorías, uchuva tipo exportación, nacional y rajada el peso del fruto sin cáliz en los diferentes tratamientos presenta diferencias altamente significativas.
Según la prueba de Student-Newman-Keuls para peso sin cáliz (tabla 3), la humedad del suelo incide en los tratamientos, siendo los tratamientos de humedad (50 a 75\%) los que produjeron el mayor peso. También Rodríguez y Pinzón (2000) encontraron el mayor peso del fruto de uchuva aplicando el polímero absorbente de agua Stockosorb en 10,20 y $30 \mathrm{~g} /$ planta $(9,8,10,0$ y 10,0 g) comparado con el testigo (7,8 g). Resultado similar obtuvo Torres et al. (2004) quienes encontraron que al aplicar mayor cantidad de agua al inicio del tratamiento se obtuvo el pico más alto de producción.

Tabla 2. Análisis de varianza del peso fresco del fruto sin cáliz.

\begin{tabular}{|l|c|c|c|c|c|}
\hline \multicolumn{1}{|c|}{ Fuente } & 9 & Suma de cuadrados & Cuadrado de la media & F-Valor & Pr $>$ F \\
\hline Modelo & 5981 & 135.537 .981 & 15.059 .776 & 19,59 & $<0,0001$ \\
\hline Error & 5990 & 4.597 .923 .723 & 0.768755 & & \\
\hline Total correcto & DF & 4.733 .461 .704 & & & \\
\hline
\end{tabular}

$\mathrm{R}^{2}=$ modelo 0,028634, Raíz cuadrado medio del error $=0,876787$. 
En el presente estudio, posiblemente la uchuva aumentó su peso considerablemente hasta la madurez fisiológica en la planta, por tanto, reaccionó con el rajado por aplicaciones tardías de agua en el desarrollo del fruto (Fischer, 2005). De los resultados anteriores se puede decir que la variable que ocasionó el rajado fue el aumento de la humedad en el suelo. Resultado similar reportaron Gordillo et al. (2004) quienes encontraron que el riego afectó los porcentajes de frutos rajados en los diferentes tratamientos, a mayor cantidad de agua mayor el rajado del fruto de uchuva. También estos resultados coinciden con los de Rodríguez y Pinzón (2000) que registraron un aumento del número de frutos de uchuva rajados por planta aplicando 20 y $30 \mathrm{~g}$ del polímero absorbente de agua Stockosorb, comparado con solamente $10 \mathrm{~g}$.

El menor porcentaje de rajado lo presentaron los tratamientos de menor humedad (10-25\%) y testigo. Al adicionar la mayor cantidad de agua (50-75\%) se evidenció el aumento del peso, el porcentaje de rajado y de la cantidad de frutos tipo exportación. El tratamiento 3,0 Calh. 50-75\% Hum., dentro de los que contenían este porcentaje de humedad (1,5 y o Calh.) obtuvo un menor porcentaje de rajado (17,67\%), este resultado se podría explicar por efecto de la alta concentración del producto comercial (3,0 L h ha $\left.{ }^{-1}\right)$.

Tabla 3. Efecto de tres niveles de humedad en el suelo sobre el peso fresco del fruto de la uchuva sin cáliz.

\begin{tabular}{|c|c|}
\hline Humedad del suelo $(\%)$ & Peso fresco fruto $(\mathrm{g})$ \\
\hline $10-25$ & $3,65 \mathrm{c}^{*}$ \\
\hline $25-50$ & $3,79 \mathrm{~b}$ \\
\hline $50-75$ & $3,97 \mathrm{a}$ \\
\hline
\end{tabular}

*Promedios con letras diferentes indican diferencias significativas según las pruebas de comparación múltiple de Student-NewmanKeuls $(P \leq 0,05)$.
La prueba de Student-Newman-Keuls para peso sin cáliz (tabla 4), muestra que el mayor peso se obtuvo con una dosis de producto de 3,0 L/ha-1 haciéndose evidente que los ácidos carboxílicos adicionados con Calcio y Boro (Calhard) deben ser suministrados en esta cantidad con el fin de aumentar el peso del fruto significativamente debido a que no se reportaron diferencias sig-

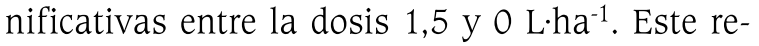
sultado coincide con lo reportado por Innovak Ltda. (2006) que investigaciones en la Universidad de Arkansas mostraron que la aplicación de ácidos polihidrocarboxílicos incrementa el flujo de carbono a través del ciclo oxidativo de las pentosas fosfato. Resultados similares encontraron Román y Gutiérrez (1998) en melón por adición de ácidos carboxílicos incrementando el peso del fruto. Cooman et al. (2005) observaron un aumentó del peso de las uchuvas cuando se aplicó Ca y B.

La prueba de Student-Newman-Keuls para peso sin cáliz (tabla 5), muestra que la dosis que produjo el mayor peso fue de 3,0 L/ha-1 combinado con humedad del 50 al $75 \%$, niveles correspondientes al tratamiento 3,0 Calh. 50-75\% Hum. lo que presentó los mejores resultados debido a que se le aplicó mayor dosis de producto y humedad del suelo. Este tratamiento aumentó el peso del fruto, obtuvo el menor porcentaje de rajado en

\section{Tabla 4. Efecto de tres dosis del producto Calhard sobre el peso fresco del fruto de la uchuva sin cáliz.}

\begin{tabular}{|c|c|}
\hline Dosis de Calhard $\left(L \cdot h a^{-1}\right)$ & Peso fresco fruto $(\mathrm{g})$ \\
\hline 0 & $3,72 \mathrm{~b}^{*}$ \\
\hline 1,5 & $3,77 \mathrm{~b}$ \\
\hline 3,0 & $3,90 \mathrm{a}$ \\
\hline
\end{tabular}

*Promedios con letras diferentes indican diferencias significativas según las pruebas de comparación múltiple de Student-NewmanKeuls $(P \leq 0,05)$. 
Tabla 5. Efecto de tres niveles de humedad en el suelo sobre el peso fresco del fruto de la uchuva sin cáliz.

\begin{tabular}{|l|c|}
\hline \multicolumn{1}{|c|}{ Tratamiento } & Peso fresco fruto $(\mathrm{g})$ \\
\hline 0 Calh. $10-25 \%$ Hum. & $3,58 \mathrm{f}^{*}$ \\
\hline 0 Calh. $25-50 \%$ Hum. & $3,75 \mathrm{de}$ \\
\hline 0 Calh. $50-75 \%$ Hum. & $3,98 \mathrm{ab}$ \\
\hline 1,5 Calh. $10-25 \%$ Hum. & $3,66 \mathrm{fe}$ \\
\hline 1,5 Calh. $25-50 \%$ Hum. & $3,75 \mathrm{de}$ \\
\hline 1,5 Calh. $50-75 \%$ Hum. & $3,89 \mathrm{dc}$ \\
\hline 3,0 Calh. $10-25 \%$ Hum. & $3,79 \mathrm{dc}$ \\
\hline 3,0 Calh. 25-50\% Hum. & $3,86 \mathrm{dc}$ \\
\hline 3,0 Calh. $50-75 \%$ Hum. & $4,04 \mathrm{a}$ \\
\hline Testigo & $3,58 \mathrm{f}$ \\
\hline
\end{tabular}

*Promedios con letras diferentes indican diferencias significativas según las pruebas de comparación múltiple de Student-NewmanKeuls $(P \leq 0,05)$.

comparación con los tratamientos 0 y 1,5 Calh. a 50-75\% Hum. Los tratamientos 0 Calh. 10-25\% Hum. y testigo produjeron el menor peso del fruto.

\section{DIÁMETRO ECUATORIAL DEL FRUTO}

El análisis de varianza para diámetro muestra una diferencia altamente significativa entre tratamientos, $\operatorname{Pr}>\mathrm{T}<0,0001$. Según la prueba de Student-Newman-Keuls para diámetro (tabla 6), la humedad del suelo incide en los tratamientos, siendo los tratamientos de humedad (50-75\%) los que produjeron el mayor diámetro. En comparación, Rodríguez y Pinzón (2000) no encontraron un aumento en el valor del diámetro del fruto de la uchuva con la aplicación del polímero absorbente de agua Stockosorb, no obstante el peso del fruto aumentaba con este producto. Posiblemente, en el ensayo de estos autores en El Rosal (Cundinamarca), los frutos tuvieron un diámetro general muy alto, entre 2,29 y 2,30 $\mathrm{mm}$, por lo cual el mejor suministro del agua no pudo agrandar más su tamaño, mientras en el presente estudio en Chocontá, los frutos fueron más pequeños y el agua pudo haber tenido un efecto más favorable en su crecimiento.

La prueba de Student-Newman-Keuls para diámetro (tabla 7), muestra que las dosis que produce el mayor diámetro son de 3,0 y 1,5 L:ha-1 ${ }^{-1}$ donde no hay diferencias significativas entre estas. Resultado contrastado con el estudio realizado por Román y Gutiérrez (1998) en melón donde la adición de ácidos carboxílicos no aumentó significativamente el diámetro del fruto.

La prueba de Student-Newman-Keuls para diámetro (tabla 8), muestra que en la combinación humedad por producto los mejores tratamientos fueron el 3,0, Calh., seguido del 0 Calh. y el 1,5 Calh., todos a 50-75\% Hum. produciendo el mayor diámetro, mientras el testigo resultó en los frutos más pequeños. Los tratamientos con contenido de alta humedad (50 a 75\%) no produjeron solamente los diámetros mas grandes, sino también el mayor porcentaje de frutos rajados, sin y con

Tabla 6. Efecto de tres niveles de humedad en el suelo sobre diámetro ecuatorial del fruto de la uchuva sin cáliz.

\begin{tabular}{|c|c|}
\hline Humedad del suelo (\%) & Diámetro fruto $(\mathrm{mm})$ \\
\hline $10-25$ & $18,22 \mathrm{c}^{*}$ \\
\hline $25-50$ & $18,50 \mathrm{~b}$ \\
\hline $50-75$ & $18,95 \mathrm{a}$ \\
\hline
\end{tabular}

*Promedios con letras diferentes indican diferencias significativas según las pruebas de comparación múltiple de Student-NewmanKeuls $(P \leq 0,05)$.

Tabla 7. Efecto de tres dosis del producto Calhard sobre el diámetro ecuatorial de la uchuva sin cáliz.

\begin{tabular}{|c|c|}
\hline $\begin{array}{c}\text { Dosis de Calhard } \\
\left(L \cdot h a^{-1}\right)\end{array}$ & Diámetro fruto $(\mathrm{mm})$ \\
\hline 0 & $18,39 \mathrm{~b}^{*}$ \\
\hline 1,5 & $18,56 \mathrm{a}$ \\
\hline 3,0 & $18,95 \mathrm{a}$ \\
\hline
\end{tabular}




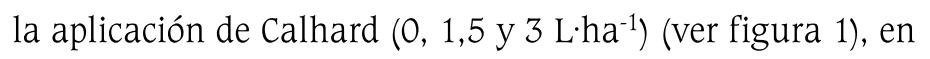
comparación con los tratamientos de menor humedad $(0 \mathrm{a}$ $25 \%)$ y testigo con un menor porcentaje de rajado.

Tabla 8. Efecto de tres dosis del producto Calhard $\left(0,1,5\right.$ y $\left.3 L^{\prime} \cdot h a^{-1}\right)$ y tres niveles de humedad en el suelo $(10-25,25-50$ y $25-75 \%)$ sobre el diámetro ecuatorial de la uchuva sin cáliz.

\begin{tabular}{|l|c|}
\hline \multicolumn{1}{|c|}{ Tratamiento } & Diámetro fruto $(\mathrm{mm})$ \\
\hline 0 Calh. $10-25 \%$ Hum. & $18,14 \mathrm{~cd}^{*}$ \\
\hline 0 Calh. $25-50 \%$ Hum. & $18,51 \mathrm{~b}$ \\
\hline 0 Calh. $50-75 \%$ Hum. & $18,95 \mathrm{a}$ \\
\hline 1,5 Calh. $10-25 \%$ Hum. & $18,33 \mathrm{~cd}$ \\
\hline 1,5 Calh. $25-50 \%$ Hum. & $18,50 \mathrm{~b}$ \\
\hline 1,5 Calh. $50-75 \%$ Hum. & $18,85 \mathrm{a}$ \\
\hline 3,0 Calh. $10-25 \%$ Hum. & $18,46 \mathrm{~b}$ \\
\hline 3,0 Calh. $25-50 \%$ Hum. & $18,49 \mathrm{~b}$ \\
\hline 3,0 Calh. $50-75 \%$ Hum. & $19,05 \mathrm{a}$ \\
\hline Testigo & $17,96 \mathrm{~d}$ \\
\hline
\end{tabular}

*Promedios con letras diferentes indican diferencias significativas según las pruebas de comparación múltiple de Student-Newman-Keuls $(P \leq 0,05)$.

\section{PESO FRESCO DEL CÁLIZ}

En el caso de exportar la uchuva con cáliz, el peso de este órgano es parte del peso del producto total (Icontec, 1999). Gálvis et al. (2005) caracterizaron el cáliz de la uchuva como el mejor empaque (natural) del fruto, siendo su perfecto estado y tamaño importante para su protección en el manipuleo poscosecha y la comercialización.

El peso fresco del cáliz osciló entre 0,55 y 0,59 g observando que el peso de este órgano aumentó con la humedad del suelo, siendo el tratamiento 3,0 Calh. 50-75\% Hum. el de mayor peso promedio, coincidiendo con el tratamiento que presentó mayor porcentaje de fruta tipo exportación.

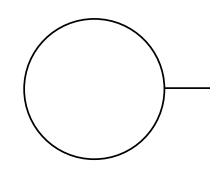

Campos, A. 2000. Manejo del riego. pp. 9-26. En: Flórez, V.J., G. Fischer y A.D. Sora (eds.). Producción, poscosecha y exportación de uchuva (Physalis peruviana L.). Unibiblos, Universidad Nacional de Colombia, Bogotá. 175 p.

Cooman, A.; C. Torres y G. Fischer. 2005. Determinación de las causas del rajado del fruto de Uchuva (Physalis peruviana L.) bajo cubierta. II. Efecto de la oferta de calcio, boro y cobre. Agron. Colomb. 23, 74-82.

Dube, S.; J. Tewari y C. Ram. 1969. Boron deficiency in Rymer apple. Progressive Hort. 1, 33-36.

Ferguson, I.B. 1979. The movement of calcium ion non vascular tissue of plant. Soil Sci. Plant Anal. 10, 217-224.

Fischer, G. 2000. Crecimiento y desarrollo. pp. 9-26. En: Flórez, V.J.; G. Fischer y A.D. Sora (eds.). Producción,

\section{REFERENCIAS BIBLIOGRÁFICAS}

poscosecha y exportación de la uchuva (Physalis peruviana L.). Unibiblos, Universidad Nacional de Colombia, Bogotá. 175 p.

Fischer, G. 2005. El problema del rajado del fruto de uchuva y su posible control. pp. 55-82. En: Fischer, G.; D. Miranda; W. Piedrahita y J. Romero (eds.). Avances en cultivo, poscosecha y exportación de la uchuva (Physalis peruviana L.) en Colombia. Unibiblos, Universidad Nacional de Colombia, Bogotá. 221 p.

Fischer, G. y P. Lüdders. 1997. Developmental changes of carbohydrates in cape gooseberry (Physalis peruviana L.) fruits in relation to the calyx and the leaves. Agron. Colomb. 14(2), 95-107.

Fischer, G. y R. Angulo C. 1999. Los frutales de clima frío en Colombia. La uchuva. Ventana al Campo Andino 2(1), 3-6. 
Gordillo, O.; G. Fischer y R. Guerrero. 2004. Efecto del riego y de la fertilización sobre la incidencia del rajado en frutos de uchuva (Physalis peruviana L.) en la zona de Silvana (Cundinamarca). Agron. Colomb. 22(1), 53-62.

Grange, R. 1993. Crecimiento del fruto. En: Azcón-Bieto, J. y M. Talón (eds.). Fisiologia y bioquímica vegetal. Interamericana McGraw-Hill, Bogota. pp. 449-462.

Herrera, A. 2000. Manejo poscosecha. pp. 109-127. En: Flórez, V.J., G. Fischer, A.D. Sora. Producción, poscosecha y exportación de la uchuva (Physalis peruviana L.). Unibiblos, Universidad Nacional de Colombia, Bogotá. 175 p.

Icontec. 1999. Frutas frescas. Uchuva. Especificaciones. Norma Técnica Colombiana NTC 4580. Instituto Colombiano de Normas Técnicas, Bogotá. 15 p.

Innovak Colombia Ltda. 2006. Comunicación personal.

Kienholz, J. 1942. Boron deficiency in pear trees. Phytopathol. 32, 1.082-1.086.

Kirk, E.R. 1962. Enciclopedia de tecnología química. Tomo 1. UTHEA, México, D.F. pp. 267-268.

Ministerio de Agricultura y Desarrollo Rural. 2006. Observatorio Agrocadenas Colombia. En: http://www.agrocadenas.gov.co/frutales/reportes; consulta: mayo 2006.

Peet, M.M. 1992. Radial fruit cracking in tomato. HortTechnol. 2(2), 216-223.

Peet, M.M. y D.H. Willits. 1995. Role of excess water in tomato fruit cracking. HortScience 30(1), 3, 65-68.

Pierson, S.F. ; M.J. Ceponis y L.P. McColloch. 1971. Market diseases of apples, pears and quinces. USDA-ARS Handbook 376.112 p.

Rease, J. 1989. Physiological disorders and maladies of pear fruit. Hort. Rev. 11, 359-407.

Rodríguez, S.F. 1992. Fertilizantes. Nutrición vegetal. AGT Editor, México, D.F.

Rodríguez, L.H. y E. Pinzón. 2000. Efecto de polímeros absorbentes "Stockosorb" y "Terracottem" en el cul- tivo de la uchuva (Physalis peruviana L.). Trabajo de grado. Facultad de Agronomía, Universidad Nacional de Colombia, Bogotá.

Rojas, G.M. y M. Rovalo. 1985. Fisiología vegetal aplicada. 3a. edición. Editorial McGraw Hill, México, D.F.

Román, M.L.F. y M.A. Gutíerrez. 1998. Evaluación de ácidos carboxílicos y nitratos de calcio para incrementar calidad, cantidad y vida de anaquel en tres tipos de melón. Rev. Soc. Mexicana Ciencia Suelo 16(1), 36-44

Salisbury, F.B. y C.W. Ross. 1994. Fisiología vegetal. Grupo Editorial Iberoamérica, México. 759 p.

Sanabria, S. 2005. Situación actual de la uchuva en Colombia. pp. 1-8. En: Fischer, G., D. Miranda, W. Piedrahita y J. Romero. 2005. Avances en cultivo, poscosecha y exportación de la uchuva (Physalis peruviana L.) en Colombia. Unibiblos, Universidad Nacional de Colombia, Bogotá. 221 p.

SAS. 1999. SAS/STAT User's guide. Version 8 Vol. 2. SAS Institute, Cary.

Shear, C.B. 1971. Symptoms of calcium deficiency on leaves and fruit of 'York Imperial' apple. J. Amer. Soc. Hort. Sci. 96, 414-417.

Street, H.E. 1969. Metabolismo de las plantas. Editorial Aihanibra, México.

Stutte, C. 1995. Laboratory evaluation of TOG NH4 on cotton, tomato, and soybean. Crop Sci. 35, 1069 1073.

Torres, C.; A. Cooman y G. Fischer 2004. Determinación de las causas del rajado del fruto de uchuva (Physalis peruviana L.) bajo cubierta. I. Efecto de la variación en el balance hídrico. Agron. Colomb. 22(2), 140-146.

Uvalle, B.J.X. 1992. Contenido y esencia de la nutrición vegetal. En: Memorias del curso de nutrición vegetal y fertilidad de suelos. Fertimex, Colegio de Postgraduados e ITSON. Obregón, Son, México.

Watkins, C.B. e I.B. Ferguson. 1981. Ions relations of apple fruit tissue during development and ripening. Calcium uptake. Soil Sci. Plant Anal. 7, 211-213. 


\section{sion Centro de Investigaciones y Asesorias Agroindustriales

\section{SERVICIOS}

\section{LABORATORIO DE FERTILIDAD DE SUELOS:}

- Análisis de suelos, agua para riego, foliares, abonos orgánicos y soluciones nutritivas.

- Recomendaciones de fertilización para cultivos en suelo e hidropónicos.

- Análisis de metales pesados en abonos orgánicos y tejido foliar.

- Análisis de fibras en pastos.

\section{LABORATORIO DE FITOPATOLOGÍA:}

- Diagnóstico de enfermedades de las plantas (hongos, bacterias, virus y nemátodos).

- Análisis de hongos y bacterias patógenas en suelo, aguas para uso agrícola e insumos orgánicos( cascarillas, compost, gallinazas, bovinazas y porquinazas).

- Evaluación de microorganismos en cuarto frío.

- Pruebas de antagonismo con hongos.

- Pruebas de eficacia de productos para el control de enfermedades en condiciones de laboratorio.

- Calidad de aguas para uso agrícola (coliformes).

- Identificación de plagas del suelo (colémbolos sinfílidos, y otros artrópodos).

\section{LABORATORIO DE ENTOMOLOGÍA:}

- Pruebas de eficacia de productos biológicos y químicos para el control de artrópodos plaga de cultivos (registro ICA).

- Venta de Controladores biológicos para mosca blanca: Encarsia formosa y Amitus fuscipenis.

- Feromonas para el control de Tuta absoluta y Neoleucinodes elegantalis.

- Asesorías en manejo integrado de plagas.

- Laboratorio de Control de Calidad de Bioinsumos y Extractos Vegetales de uso Agrícola (único en Colombia con Registro ICA).

\section{PROPAGACIÓN DE PLANTAS:}

- Producción tecnificada de plántulas de hortalizas, hierbas aromáticas, frutales de clima frío y frío moderado y ornamentales.

- Evaluación de híbridos y variedades de hortalizas, aromáticas, frutales y ornamentales.
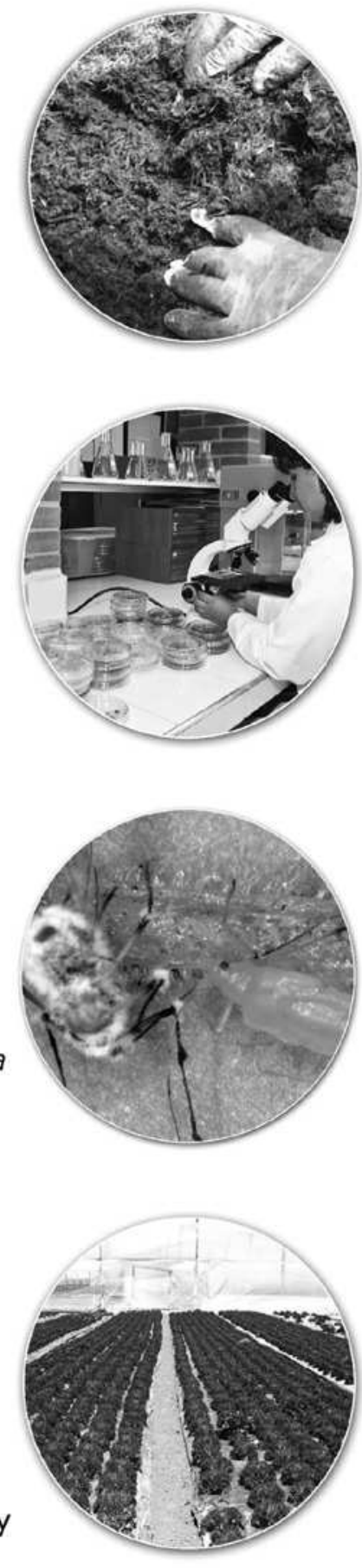

\section{CAPACITACIÓN Y PUBLICACIONES}

Carretera Central del Norte 3 kilómetros adelante de La Caro, Chía, Cundinamarca. Teléfonos: 8650218/219/239/127 